\title{
Identification of ferroptosis-related subtypes, characteristics of TME infiltration and development of prognostic models in gastric cancer
}

RUI LING ( $\sim$ lingruiray@163.com )

Affiliated Hospital of Jiangsu University

XIANG TANG

Affiliated Hospital of Jiangsu University

NA LIU

Affiliated Hospital of Jiangsu University

YUNPENG YU

Affiliated Hospital of Jiangsu University

YUTING SU

Affiliated Hospital of Jiangsu University

YUEPENG ZHOU

Affiliated Hospital of Jiangsu University

DEYU CHEN

Affiliated Hospital of Jiangsu University

\section{Research Article}

Keywords: Ferroptosis, gastric cancer, tumor microenvironment, microsatellite instability, cancer stem cell, overall survival

Posted Date: March 16th, 2022

DOI: https://doi.org/10.21203/rs.3.rs-1377620/v2

License: (a) (1) This work is licensed under a Creative Commons Attribution 4.0 International License.

Read Full License 


\section{Abstract}

Ferroptosis constitutes a novel type of cell death in morphology, biochemistry and genetics, and is tightly associated with the occurrence, development, prognosis and treatment of tumors. Nevertheless, the potential contribution of ferroptosis-related genes (FRGs) to the tumor microenvironment (TME) is indistinct. We delineated and evaluated the expression pattern of FRGs in gastric cancer (GC) samples from the perspectives of genetics and transcription. Subsequently, we discerned two different molecular subtypes and figured out that the changes in multilayer FRGs are linked with the clinicopathological characteristics, prognosis and TME cell infiltration characteristics of patients. Then, we constructed an FRG-score with a view to predicting overall survival (OS) and verified its predictive ability in GC patients. Therefore, we constructed a high-precision nomogram to improve the clinical applicability of the FRGscore. Low FRG-score, due to its high microsatellite instability (MSI-H), high mutational load and immune activation, indicates the possible advantage of OS. In addition, the FRG-score was closely related to the cancer stem cell (CSC) index and the sensitive degree of chemotherapeutic drug. Our generalized study of FRGs in gastric cancer shows their potentialities in the TME, clinicopathological characteristics and prognosis. These results may advance the existing knowledge of FRGs in gastric cancer, and push ahead with more effective prognostic assessment and the development of more effective immunotherapy approaches.

\section{Introduction}

Ferroptosis is a new-fangled kind of cell death that has been discovered in the fields of morphology, biochemistry, and genetics. and was first proposed by Dixon in 2012. It mainly involves lipid peroxidation, iron ion metabolism and the system Xct-GSH-GPX4 axis [1]. Ferroptosis results from the weakening of glutathione-dependent antioxidant defense capabilities, bringing about uninhibited lipid peroxidation and ultimately cell death [2]. Neither such apoptosis inhibitors as necrostatin-1 nor autophagy inhibitors including chloroquine have been shown to prevent ferroptosis from occurring.[1], but it is able to be inhibited by such iron chelating agents as deferoxamine or such synthetic antioxidants as ferrostatin-1 [3]. Ferroptosis contributes significantly to the occurrence and growth of many cancers, including gastric carcinoma (GC) $[4,5]$. As an epithelial malignant tumor, gastric cancer (GC) is the third greatest contributor to cancer mortality worldwide, occupying seven percent of all cancer diagnoses and nine percent of total cancer deaths [6]. According to cancer statistics published by the Chinese government, GC accounts for the second most regular malignant tumor in China; particularly, stomach adenocarcinoma (STAD) is the most common in GC [6]. Apatinib can induce lipid peroxidation through SREBP-1a-mediated GPX4 and then negatively regulate ferroptosis, GC cells, and even multidrug-resistant GC cells [7]. Cysteine dioxygenase type 1 (cysteine dioxygenase type 1, CD01) mediates erastin-induced ferroptosis of AGS and BGC823 gastric cancer cells [8].

Increasing evidence shows the close connection between ferroptosis and the tumor immune microenvironment (TME) $[9,10]$. It is commonly believed that the tumor microenvironment (TME) has a role in the formation and progression of malignancies [12]. Tumor cells are just one component of the 
tumor microenvironment (TME), which also includes fibroblasts, immunological and inflammatory cells, endothelial cells, extracellular matrix components, and cancer-related cytokines and chemokines. [13] Tumor cells interact with these components, resulting in the production of tumor microenvironment (TME), which has an impact on the genesis and progression of cancer. Infiltrating immune cells (TIICs) in the tumor microenvironment (TME) have been shown to be able to predict the prognosis of cancer. Most studies only analyze one or two ferroptosis-related genes (FRGs) and cell types because to technological restrictions. This is because the antitumor impact is defined by numerous genes interacting in a highly coordinated way. As a result, a comprehensive understanding of the characteristics of TME cell infiltration mediated by multiple FRGs has the potential to provide meaningful insights into the mechanisms of GC tumorigenesis and prediction of immunotherapy response.

This study comprehensively assessed the expression profile of FRGs, and used two calculation algorithms (CIBERSORT and ESTIMATE) to obtain a comprehensive understanding of the immune landscape within the tumor. First, GC patients were divided into two discrete subtypes based on the expression level of FRGs. According to the differentially expressed genes (DEGs) identified by the two subtypes of ferroptosis, patients were divided into two gene subtypes. We further developed a scoring system to predict overall survival (OS) and characterize the immune landscape of GC to accurately predict the patient's prognosis and response to immunotherapy.

\section{Materials And Methods}

\section{Patients and tumor tissues}

Patients with GC who had not had chemotherapy or radiation, had given informed permission before surgical resection, and the data gathered from 2020 to 2021 at the Affiliated Hospital of Jiangsu University were used to create a cohort of 10 tumors and para-carcinoma tissues (Zhenjiang, China). Qualified pathologists validated all tissue samples, which were then stored in liquid nitrogen and preserved at $-80^{\circ} \mathrm{C}$ until utilized for qRT-PCR. The Affiliated Hospital of Jiangsu University's Research Ethics Committees authorized this research, and all patients provided written informed permission.

\section{Quantitative Real-time PCR analysis}

In accordance with the manufacturer guidelines, total RNA was separated from human colon cancer tissues and cells by virtue of the TRIzol reagent (Takara Biotechnology Company, Ltd.). The Bio Mate $3 S$ Analyzer was used to determine the relative levels of RNA in the samples (Thermo Fisher Scientific, San Jose, CA, USA). Next, the qRT-PCR reaction was conducted by a reverse transcription reagent kit (Vazyme Biotech Co., Ltd). and Cham Q SYBR qPCR Master mix (Vazyme Biotech Co. Ltd.) in Mx3000P system (Agilent, CA, USA). The primers were as listed in Table S3. Relative expression levels were worked out using the $2-\Delta \Delta \mathrm{Cq}$ method and $\beta$-Actin was adopted as an internal reference gene. The experiments were repeated three times in triplicate. Data sources 
Gene Expression (fragments per kilobase million, FPKM)as well as associated prognostic and clinicopathological data, were gained from the Cancer Genome Atlas (TCGA) (https://portal.gdc.cancer) and Gene Expression Comprehensive Database (GEO) (https://www.ncbi.nlm.nih.gov/geo/) databases. TCGA-STAD cohort and GEO-GSE84437 cohort were obtained for further examination. The FPKM value of TCGA-STAD was converted into one million transcripts per kilobase (TPM) and was considered to be the same as the transcript in the microarray. TCGA and GEO datasets were combined by using the "limma" and "Sva" packages in R. Detailed information about GC patients is presented in Table S1.

\section{Consensus clustering analysis of FRGs}

FRGs were acquired from the FerrDb database (http://www.zhounan.org/ferrdb). Table S2 has further data on these genes, as well as a list of their features. Consensus unsupervised cluster analysis was conducted using the R package "ConsensusClusteringPlus," and patients were categorized into distinct molecular subtypes based on the expression of FRG. Following that, principal component analysis (PCA) was carried out with the help of the "ggplot2" R package.

\section{Relationship between molecular subtypes and prognosis and TME of GC}

First, We utilized the Kaplan-Meier curve produced by the "survival" and "survivor" R packages to assess the difference in overall survival (OS) across various subtypes of cancer. Second, the immune and matrix scores of each patient were evaluated using ESTIMATE algorithms.

\section{DEG identification}

When the "limma" package in $\mathrm{R}$ was ultilized to find the DEGs between various subtypes, the fold change was 1.5 and the altered $p$ value was less than 0.05 , indicating a significant difference.

\section{Construction of the ferroptosis-related prognostic FRG-score}

The ferroptosis pattern of a single tumor was quantified by calculating the ferroptosis score. First, a single-factor The DEGs were under Cox regression analysis in order to find the genes associated with OS. For the purpose of a more in-depth study, patients were allocated into two subclass groupings (ferroptosis gene subtype A and ferroptosis gene subtype B); the unsupervised clustering approach on the basis of the expression of prognostic FRGs was employed to categorize them. In the end, all GC patients were separated into training and testing categories using a random number generator, and the results of the former were utilized to calculate the ferroptosis-related prognostic FRG-score. It was decided to utilize the Lasso Cox regression approach, which was based on prognostic genes associated with ferroptosis. The "glmnet" R package was adopted to reduce the danger of overfitting the model. Multivariate Cox analysis was used to select candidate genes, and the prognostic FRG-score was established in the training set. The calculation method of FRG-score is as follows: FRG-score $=\Sigma$ (Expi * coefi), The risk coefficient and gene expression level of each gene are represented by the variables coefi and Expi, respectively. 


\section{Formation and verification of the nomogram scoring system}

Using clinical features and risk scores and taking root in the results of independent prognostic analysis, the "rms" software package was expected to foster a predictive nomogram. The nomogram was evaluated by time-dependent receiver operating characteristic (ROC) curves of three-year, five-year, and ten-year survival rates. The nomogram's calibration chart was used to characterize the expected values between the predicted three-year, five-year, and ten-year survival events and the results actually observed.

\section{Assessment of the immune status, microsatellite instability (MSI) and cancer stem cell (CSC) index between the high- and low- risk groups}

To assess the proportion of TIICs in the TME, ssGSVA was adopted to detect the abundance of infiltrating immune cells from the low-risk and high-risk groups. We also made use of the ESTIMATE package to evaluate the TME scores (stromal score, immune score, and estimated score) of the two groups. We then investigated the relationship between the infiltrating immune cells and eight genes in the FRG-score by CIBERSORT. In addition, we utilized box plots to examine if there was any discrepancy in the expression levels of immunological checkpoints across the groups. Moreover, the association between these two risk categories and the MSI and CSC scores as well were looked at.

\section{Gene mutation and drug sensitivity analysis}

To identify the somatic mutations in GC patients, we used the "maftools" R package to generate the mutation annotation format (MAF) in the TCGA database. We also assessed the tumor mutation burden (TMB) score in each GC patient. To explore the difference in the efficacy of chemotherapy drugs between the two groups of patients, we used the "pRRophetic" package to calculate the half inhibitory concentration (IC50) value of the commonly applied chemotherapy drugs for the treatment of GC.

\section{Statistical analyses}

All statistical analyses were carried out under the aegis of $\mathrm{R}$ version 4.0.5. All the results were considered significant with $p<.05$.

\section{Results}

\section{Genetic and transcriptional changes in of FRGs in gastric cancer}

Totally, 44 FRGs participated in this investigation. When the incidence of somatic mutations in these FRGs was investigated, it was discovered that of 433 GC samples, 252 (58.2\%) had FRG mutations. In the GC cohort, TP53 has the highest mutation frequency (44\%) (Figure 1A). Since the results showed that TP53 had the highest mutation frequency, we went on to look into the correlation between the TP53 mutation and FRG expression in more detail." TP53 mutation status was shown to be highly linked with the expression levels of 11 FRGs, according to the findings of the study (Figure 1B). Following that, we looked into the somatic copy number changes that occurred in these FRGs and found that copy 
number changes occurred across all 44 FRGs. Among them, ACSF2, HSPA5, TFR2, BECN1 and HMGB1 copy number variation (CNV) generally increased, while CD01, SCD, GOT1, CHAC1 and PLIN4 CNV generally decreased (Figure 1C). The location of CNV changes on each chromosome is depicted in Figure 1D. We next analyzed the levels of mRNA expression between normal tissues and GC, and discovered that the expression levels of the majority of FRGs were positively linked with changes in CNV. The expression level of FRGs with CNV gain, including HSPA5, TFR2 and BECN1, was considerably higher in GC samples than in normal stomach samples, while the expression levels of FRGs with CNV deletions, including CDO1 and PLIN4, were significantly decreased in GC samples (Fig. 1E), reflecting that mRNA expression may be regulated by CNVs. Yet, some FRGs with CNV deletions, including SCD, exhibited up-regulated mRNA expression, whereas other FRGs with high CNV gain or deletion frequencies did not differ between GC samples and normal samples. Therefore, while CNV may make clear many of the alterations observed in FRG expression, it is not the sole determinant regulating mRNA expression. Other factors, such as epigenetic regulation [11], transcription factors and regulation of the interaction between transcription factors and transcription mechanisms [12,13], can also regulate gene expression. Our analysis results show that there are significant differences in the genetic landscape and expression level of FRGs between $\mathrm{GC}$ and control samples, demonstrating the potential contribution of FRGs to GC tumorigenesis.

\section{Identification of ferroptosis subtypes of gastric cancer}

To thoroughly apprehend the expression pattern of FRGs involved in tumorigenesis, we included 871 patients from two stomach cohorts (TCGA-STAD, GSE84437) in the study for in-depth exploration. Table S1 includes details for roughly $871 \mathrm{GC}$ cases, with a total of 871 patients. In the light of the outcomes of the univariate Cox regression and Kaplan-Meier analysis (Table S4), 11 out of the 44 FRGs had prognostic significance (p. 05), which was utilized as the filtering criteria. Following that, we carried out a multivariate Cox regression analysis on 11 prognostic FRGs, three of which (HMGB1, KEAP, and AIFM2) were found as independent predictors (see Additional file 1). (Table S5). In a ferroptosis network, it was verified that the overall situation of FRG interactions, regulator linkages, and their predictive relevance in GC patients was confirmed (Figure 2a). A consensus clustering technique was adopted to categorize patients with GC into groups in line with the expression profiles of 44 FRGs in order to further investigate the expression properties of these proteins. According to our findings, the number $k=2$ seems to be the most appropriate number to partition the whole cohort into two subtypes, A and B. (Figure 2B). There were statistically significant variations in the ferroptosis transcription patterns between the two subtypes, as shown by PCA (Figure 2C). In accordance with the Kaplan-Meier curve, the overall survival time of patients with subtype A discernibly exceeded that of patients with subtype B.(Figure 2D). In addition, comparing the clinicopathological characteristics of different subtypes of GC, it was found that compared with cluster $B$, cluster A was preferentially associated with fewer regional lymph nodes $(p<$. 05).

\section{Characteristics of TME in different subtypes}


Recent studies suggest that ferroptosis has close relation with the tumor microenvironment $[10,14]$. To study the role of FRGs in the TME of GC, we used ssGSEA to evaluate the correlation between the two subtypes and 23 human immune cell subgroups. We perceived a notable difference in the infiltration of most immune cells between the A and B subtypes (Figure 3A). Activated CD4 T cell, gamma delta T cell, neutrophilna, type $17 \mathrm{~T}$ helper cell, type $2 \mathrm{~T}$ helper cell were significantly higher than that of subtype $\mathrm{B}$, while activated B cell, eosinophilna, immature B cell, mast cell, plasmacytoid dendritic cell, $T$ follicular helper cell and type $1 \mathrm{~T}$ helper cell were significantly lower than that of subtype B. We also used the ESTIMATE package to valued the TME scores (stromal score, immune score, and estimated score) of the two subtypes. Specifically, in terms of TME score, it is believed that the higher the stromal score or immunological score, the greater the relative content of stromal cells or immune cells in the TME, and that the estimated score shows the aggregation of stromal cells or immune scores in this environment. The outcomes show that patients with subtype A have higher TME scores (Figure 3B). In addition, we evaluated some important immune checkpoints such as CTLA4, PDCD1, CD274, LAG3, PDCD1LG2 and HAVCR2, and compared to cluster B, these immune checkpoints had higher expression in cluster A (Figure $3 \mathrm{C}-3 \mathrm{H})$.

\section{Gene subtype identification based on DEGs}

To study the underlying biological behavior of each ferroptosis mode, we used the R package "limma" to identify 695 ferroptosis subtype-related DEGs (Figure 4A-4B; Table S6). KEGG analysis showed the enrichment of cancer-related pathways (Figure 4B), demonstrating that ferroptosis functions critically in the immunological control of the transmembrane environment. For further validation of this regulatory mechanism, we employed a consensus clustering algorithm to categorize patients with GC into two gene subtypes, $A$ and $B$, using a consensus clustering technique (Figure $4 \mathrm{C}$ ). Aside from that, we performed a univariate Cox regression analysis to determine the prognostic value of 695 subtype-related genes, and screened 370 genes associated with overall survival time (p.05) for further investigation in the future (Table S7). Patients with the $B$ gene subtype had the poorest OS, while patients with the $B$ gene cluster had the best OS, according to Kaplan-Meier curves (Figure 4D). Additionally, ferroptosis gene subtype B patterns were linked with higher regional lymph nodes (Figure 4E). Further, we made a comparison of the expression levels of FRGs mRNAs between gene cluster $A$ and $B$, and found that the expression levels of most FRGs were drastically diverse (Figure 4F).

\section{Construction and verification of prognostic FRG-score}

We developed FRG ratings that were based on DEGs associated with subtypes The distribution of patients with two subtypes of ferroptosis, two subtypes of genotypes, and two groups of FRG-scores are shown in Figure 5A. First, we randomly allocated patients into training and test groups in a 1:1 ratio using the "caret package" in R. We conducted Lasso and multivariate Cox analyses of 370 prognostic DEGs associated with ferroptosis to further select the best prognostic features. Lasso regression analysis was then performed and 13 OS-related genes were retained based on the smallest partial likelihood bias (Fig. S5A-B). We also conducted multivariate Cox regression analysis on 20 OS-related genes. According to 
Akaike Information Criteria (AIC) values, we ultimately obtained 8 genes, including 5 high-risk genes (AKR1C2, MMRN1, NPTX1, MMP11, and KRT80) and 3 low-risk genes (GAD1, SLC27A2 and GBP5) (Figure 5B). The FRG-score was developed in accordance with the findings of multiple Cox regression analysis. It is calculated as follows: Risk score $=\left(0.0931^{\star}\right.$ expression of AKR1C2 $)+\left(0.1505^{\star}\right.$ expression of MMRN1 $)+\left(-0.1572^{\star}\right.$ expression of GAD1 $)+\left(-0.1325^{\star}\right.$ expression of SLC27A2 $)+\left(0.1202^{\star}\right.$ expression of NPTX1 $)+\left(-0.1165^{*}\right.$ expression of GBP5 $)+(0.1371 *$ expression of MMP11 $)+(0.1361 *$ expression of KRT80). Subsequently, we found significant differences in FRG-score between ferroptosis gene subtypes and ferroptosis subtypes. Figures 5C-5D showed the distribution of risk scores for the two subtypes. Patients with a FRG-score less than or equal to the median risk score were categorized as being in the lowest quartile, while patients with a FRG-score more than or equal to the median risk score were classified as being in the high-risk category.The FRG-score risk distribution chart shows that with the increase in FRG-scores, the survival time decreases and the mortality rate increases (Figure 5E-5F). We then further compared the expression levels of FRGs mRNA between FRG-score, and found that the expression levels of most FRGs had huge differences (Figure 4G). The Kaplan-Meier survival curve showed that compared with patients with high scores, patients with lower scores had significantly higher overall survival ( $p<.001$; Figure $5 \mathrm{H})$. In addition, the AUC values of the three-, five- and ten-year survival rates of the FRG-score were $0.676,0.705$ and 0.735 , respectively (Figure $5 \mathrm{I}$ ). Considering the inconvenient clinical utility of the FRG-score in predicting the OS of patients with stomach cancer, we established a nomogram containing the FRG-score and clinicopathological parameters to expect the OS rate at 3,5 and 10 years (Figure $5 \mathrm{~J}$ ). The subsequent calibration chart shows that compared with the ideal model (Figure $5 K)$.

\section{Validation of expression levels of eight FRGs for prognostic features}

RT-qPCR was used to identify the expression levels of eight prognostic genes in $10 \mathrm{GC}$ tumors and ten neighboring normal tissues. As illustrated in Figure S1, the expression levels of KRT80, GBP5, MMP11, MMRN1 and SLC27A2 were elevated in GC tissues, whereas AKR1C2, NPTX1 and GAD1 were downregulated in $\mathrm{GC}$ tissues.

\section{Assess TME and checkpoints between high-risk and low-risk group}

The relationship between the FRG-score and the number of immune cells was next investigated. As demonstrated in the scatter diagram (Figure 6A), FRG-score is positively correlated with B cell memory, B cell naïve, Mast cells resting, M2 Macrophages, Monocytes, T cells regulatory, and T cells CD4 memory resting, however, M0 Macrophages, M1 Macrophages, Neutrophils, NK cells resting, Plasma cells, CD8 ${ }^{+} \mathrm{T}$ cells, follicular helper T cells, activated memory CD4+ T cells, were negatively correlated with FRG-score. Low FRG-score is also closely related to high immune score, while high FRG-score is closely related to high matrix score (Figure 6B). Besides, we probed into the relevance between eight genes in the model and the number of immune cells present. We discovered that these eight genes were highly connected with the majority of immune cells (Figure $6 \mathrm{C}$ ). A further investigation into the correlation between immune 
checkpoints and our risk model revealed that 47 immune checkpoints were differently expressed between the two groups, as seen in Figure 6D.

\section{Relationship of FRG-score with MSI and CSC index}

According to the available information, people with high microsatellite instability (MSI-H) are more susceptible to immunotherapy and may profit from immunotherapy treatments. [15, 16]. According to correlation analysis, low FRG-scores were significantly associated with MSI-H status, while high FRGscores were significantly associated with microsatellite stable (MSS) status (Figure 7A-7B). Moreover, we incorporated the FRG-score and CSC index data in order to evaluate the possibility of the connection between FRG-score and CSC in GC. The linear correlation result between FRG-score and GC index is shown in Figure 7C. We believe that the FRG-score is negatively related to the index $(R=-0.6, p<.001)$, showing GC cells with lower FRG-scores have more evident stem cell features and exhibit lower cell differentiation, suggesting that they are more stem cell-like and less differentiated.

\section{Mutation and drug susceptibility analysis}

Patients with a high TMB may gain from immunotherapy as a result of their increased neoantigens, according to accumulating research. $[17,18]$. We analyzed mutational data from the TCGA-STAD cohort, and because the data in Figure 7 showed that TMB in the high-risk group was inferior than that in the lowrisk group, it is possible that immunotherapy might be beneficial in the low-risk group. Following the results of the Spearman correlation analysis, FRG scores were adversely connected with TMB (Figure 7E). The distribution of somatic mutations in the TCGA-STAD cohort was then compared between the two FRG score groups, which was subsequently evaluated. The top 10 mutations in the high-risk and low-risk group were TTN, TP53, MUC16, ARIDI1, LRP1B, SYNE1, FLG, CSMD3, PCLO and DNAH5 (Figure 7F-7G). Next, select chemotherapeutic treatments that are presently in use for treatment were investigated for sensitivity in individuals who were classified as low-risk or high-risk to these medications. Intriguingly, we discovered that the half-inhibitory concentrations of dasatinib and pazopanib in the high FRG-score group was lower, while the half-inhibitory concentration valuesof chemotherapeutics such as doxorubicin, gemcitabine, cisplatin, camptothecin, mitomycin-C and paclitaxel were lower in the low FRG-score group. Briefly summarized, these findings demonstrate that FRGs were associated with medication sensitivity (Figure 7H-70).

\section{Discussion}

Ferroptosis has been shown to be essential in the treatment of cancer and immunotherapy, according to a large number of studies. Although most researches have concentrated on one or two FRGs or a single kind of TME cell, the features of TME infiltration and the overall impact of numerous FRGs acting together have not been thoroughly explained to yet. The findings of this investigation demonstrated that the transcriptional and genetic levels of FRGs in GC had changed significantly. We recognized two different molecular subtypes ( $A$ and $B$ ) from 44 FRGs. Compared with patients with subtype A, patients with subtype $B$ have worse OS. In addition, there were huge differences in immune cell invasion, immune 
checkpoint, and TME characteristics between the two subtypes. In addition, the difference in the mRNA transcriptome between different ferroptosis subtypes is greatly associated with the FRG and tumorrelated pathways. Subsequently, according to the DEGs between the two ferroptosis subtypes, we identified two genotypes ( $A$ and $B$ ). Our findings suggest that FRGs can be used as predictors for assessing clinical outcomes and immunotherapy response in GC. We therefore constructed a robust and valid prognostic FRG score, displayed its predictive ability, and formed a quantitative nomogram to improve performance to a greater extent. Also, the expression levels of eight genes, including FRG-score, were investigated in gastric cancer tissue samples. FRG scores were lower and higher in ferroptosis patterns indicated by immune activation compared to those characterized by immunosuppression, respectively. Patients with low-risk and high-risk FRG scores had substantially diverse clinicopathological features, prognosis, TME, immunological checkpoints, mutations, MSI, CSC index, and treatment sensitivity than patients with a normal or high-risk FRG score, respectively. This prognostic model can be adopted to stratify the prognosis of gastric cancer patients, which will help to better understand the molecular mechanism of gastric cancer and further deliver new strategies for tumor-targeted therapy.

After conventional chemotherapy, gastric cancer has a poor prognosis due to tumor immune cell invasion and increased immune checkpoint levels. Although huge progress has been made in tumor immunotherapy, the prognosis of gastric cancer patients still shows high heterogeneity, which further highlights the key role of the TME in the occurrence and growth of gastric cancer. Immune cells in the TME participate in various immune responses and activities to promote tumor survival. The TME surrounding tumor cells includes invasive immune cells, tumor fibroblasts, and extracellular matrix. There is also evidence that the TME has a significant impact on the drug resistance of tumor treatments. In this study, the ferroptosis pattern featured by immune activation (subtype A) was related to a lower FRGscore, and the pattern featured by immunosuppression (subtype B) was correlated with higher FRG-score. We found that there were significant differences in the TME characteristics and the relative abundance of 23 TIICs between the two molecular subtypes and different FRG-scores. This finding shows that FRGs play a crucial role in the growth of gastric cancer. Increasing evidence shows that memory T cells, effector T cells, and T cell differentiation contribute vitally to the immune defense of gastric cancer. The density of tumor infiltrating $T$ cells in gastric cancer tissues is higher than that in normal tissues, demonstrating a good prognosis [19]. The increase in the proportion of gamma delta T cells in peripheral blood can be antitumor immunity and anti-gastric cancer [20]. Among the two subtypes and FRG-score, subtype $A$ and low FRG-score have a better prognosis and higher infiltration of activated memory CD4+ and gamma delta T cells, reflecting that they functions actively in the development of gastric cancer. Studies have shown that the infiltration of Tregs that inhibit the anticancer immune response has relation to poor prognosis. More Tregs existed in the TME of patients with subtype B and high FRG-scores.

The in-depth research on immunotherapy has opened up a new way for tumor treatment. Such immunotherapy involves immune checkpoint inhibitors and cell therapy. Immune checkpoint inhibitors have been studied for CTLA-4, PD-1, PD-L1 and LAG3, and their safety and effectiveness have been proven by clinical studies. Immunosuppressive agents are also used in the treatment of gastric cancer. In this study, we observed that CTLA-4, PD-1, PD-L1 and LAG3 had higher expression levels in the A subtype 
and low FRG-score groups. MSI-H is a subtype with poor survival in gastric cancer [21]. In the research, the proportion of MSI-H patients with poor prognosis A subtype and low FRG-score group was higher. Our conclusion is that patients with low FRG-scores; CTLA-4, PD-1, PD-L1, LAG3 and MSI-H expression levels may tend to have a response to immune therapy.

However, this study still has some limitations. For starters, we analyzed data from publicly available sources, and all of the samples we utilized were collected retrospectively. For this reason, further prospective studies as well as experimental investigations in vitro and in vivo are required to further verify our results in the future. In addition, there are some key clinical factors, such as surgery, neoadjuvant chemoradiotherapy and other data that cannot be analyzed, which may affect the prognosis and prognostic status of immune response.

\section{Declarations}

\section{Data availability}

Data and materials are available from corresponding authors on reasonable request.

\section{Authors' contribution}

$\mathrm{DC}$ and $\mathrm{RL}$ conceived and planned the study. RL and $\mathrm{YY}$ designed the experiments. $\mathrm{XT}, \mathrm{NL}$ and $\mathrm{YS}$ conducted the experiments and scrutinize the data. RL wrote, edited and revised the manuscript. The final manuscript has been read and approved by all the authors.

\section{Funding}

The present study was funded by The National Natural Science Foundation of China (grant no. 81572956), The Jiangsu Provincial Science and Technology Supporting Program (grant no. BE2017096), The Medical Innovation Team of Jiangsu Province (grant no. CXTDC2016009) and The Student Innovation Training Program Projects of Jiangsu University (grant no. 201810299262W).

\section{Conflict of interest}

The authors have declared no conflict of interests.

Consent to participate Written consent to participate was obtained for enrolled subjects

\section{Ethical approval}

The principles outlined in the Helsinki Declaration of 1975, as revised in 1983, have been followed for all the patients included. The protocols of this study were approved by the Ethic Committee of Affiliated Hospital of Jiangsu University.

\section{References}


1. Dixon SJ, Lemberg KM, Lamprecht MR, Skouta R, Zaitsev EM, Gleason CE, Patel DN, Bauer AJ, Cantley AM, Yang WS et al: Ferroptosis: an iron-dependent form of nonapoptotic cell death. Cell 2012, 149(5):1060-1072.

2. Cao JY, Dixon SJ: Mechanisms of ferroptosis. Cellular and molecular life sciences : CMLS2016, 73(11-12):2195-2209.

3. Dolma S, Lessnick SL, Hahn WC, Stockwell BR: Identification of genotype-selective antitumor agents using synthetic lethal chemical screening in engineered human tumor cells. Cancer cell 2003, 3(3):285-296.

4. Zhang H, Deng T, Liu R, Ning T, Yang H, Liu D, Zhang Q, Lin D, Ge S, Bai M et al: CAF secreted miR522 suppresses ferroptosis and promotes acquired chemo-resistance in gastric cancer. Molecular cancer 2020, 19(1):43.

5. Lee JY, Nam M, Son HY, Hyun K, Jang SY, Kim JW, Kim MW, Jung Y, Jang E, Yoon SJ et al: Polyunsaturated fatty acid biosynthesis pathway determines ferroptosis sensitivity in gastric cancer. Proceedings of the National Academy of Sciences of the United States of America 2020, 117(51):3243332442.

6. Chen W, Zheng R, Zuo T, Zeng H, Zhang S, He J: National cancer incidence and mortality in China, 2012. Chinese journal of cancer research = Chung-kuo yen cheng yen chiu 2016, 28(1):1-11.

7. Zhao L, Peng Y, He S, Li R, Wang Z, Huang J, Lei X, Li G, Ma Q: Apatinib induced ferroptosis by lipid peroxidation in gastric cancer. Gastric cancer : official journal of the International Gastric Cancer Association and the Japanese Gastric Cancer Association 2021, 24(3):642-654.

8. Hao S, Yu J, He W, Huang Q, Zhao Y, Liang B, Zhang S, Wen Z, Dong S, Rao J et al: Cysteine Dioxygenase 1 Mediates Erastin-Induced Ferroptosis in Human Gastric Cancer Cells. Neoplasia (New York, NY) 2017, 19(12):1022-1032.

9. Liu Y, Duan C, Dai R, Zeng Y: Ferroptosis-mediated Crosstalk in the Tumor Microenvironment Implicated in Cancer Progression and Therapy. Frontiers in cell and developmental biology 2021, 9:739392.

10. $\mathrm{Xu} \mathrm{H}, \mathrm{Ye} \mathrm{D}$, Ren $\mathrm{M}$, Zhang $\mathrm{H}, \mathrm{Bi} \mathrm{F}$ : Ferroptosis in the tumor microenvironment: perspectives for immunotherapy. Trends in molecular medicine 2021, 27(9):856-867.

11. Hogg SJ, Beavis PA, Dawson MA, Johnstone RW: Targeting the epigenetic regulation of antitumour immunity. Nature reviews Drug discovery 2020, 19(11):776-800.

12. Hill K: Post-translational modifications of hormone-responsive transcription factors: the next level of regulation. Journal of experimental botany 2015, 66(16):4933-4945. 
13. Francois $\mathrm{M}$, Donovan $\mathrm{P}$, Fontaine F: Modulating transcription factor activity: Interfering with protein-protein interaction networks. Seminars in cell \& developmental biology 2020, 99:12-19.

14. Wang Y, Wei Z, Pan K, Li J, Chen Q: The function and mechanism of ferroptosis in cancer. Apoptosis : an international journal on programmed cell death 2020, 25(11-12):786-798.

15. Zhao P, Li L, Jiang X, Li Q: Mismatch repair deficiency/microsatellite instability-high as a predictor for anti-PD-1/PD-L1 immunotherapy efficacy. Journal of hematology \& oncology 2019, 12(1):54.

16. Chang L, Chang M, Chang HM, Chang F: Microsatellite Instability: A Predictive Biomarker for Cancer Immunotherapy. Applied immunohistochemistry \& molecular morphology : AIMM 2018, 26(2):e15-e21.

17. Liu L, Bai X, Wang J, Tang XR, Wu DH, Du SS, Du XJ, Zhang YW, Zhu HB, Fang Y et al: Combination of TMB and CNA Stratifies Prognostic and Predictive Responses to Immunotherapy Across Metastatic Cancer. Clinical cancer research : an official journal of the American Association for Cancer Research 2019, 25(24):7413-7423.

18. Luchini C, Bibeau F, Ligtenberg MJL, Singh N, Nottegar A, Bosse T, Miller R, Riaz N, Douillard JY, Andre $\mathrm{F}$ et al: ESMO recommendations on microsatellite instability testing for immunotherapy in cancer, and its relationship with PD-1/PD-L1 expression and tumour mutational burden: a systematic reviewbased approach. Annals of oncology : official journal of the European Society for Medical Oncology 2019, 30(8):1232-1243.

19. Li F, Sun Y, Huang J, Xu W, Liu J, Yuan Z: CD4/CD8 + T cells, DC subsets, Foxp3, and IDO expression are predictive indictors of gastric cancer prognosis. Cancer medicine 2019, 8(17):7330-7344.

20. Lee AJ, Kim SG, Chae HD, Lee GH, Shin IH: $ү \delta$ T cells are increased in the peripheral blood of patients with gastric cancer. Clinica chimica acta; international journal of clinical chemistry 2012, 413(1920):1495-1499.

21. Yang Y, Shi Z, Bai R, Hu W: Heterogeneity of MSI-H gastric cancer identifies a subtype with worse survival. Journal of medical genetics 2021, 58(1):12-19.

\section{Figures}


A

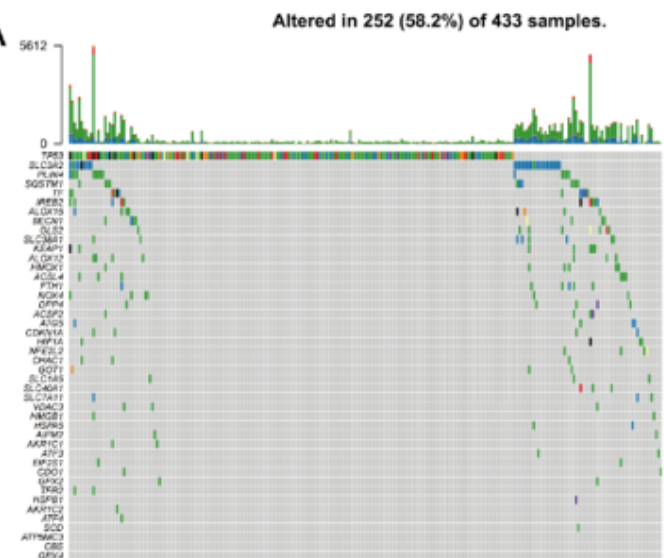

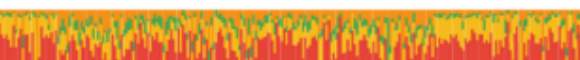

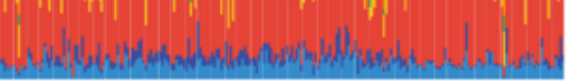

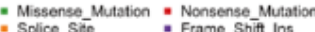

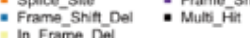

B
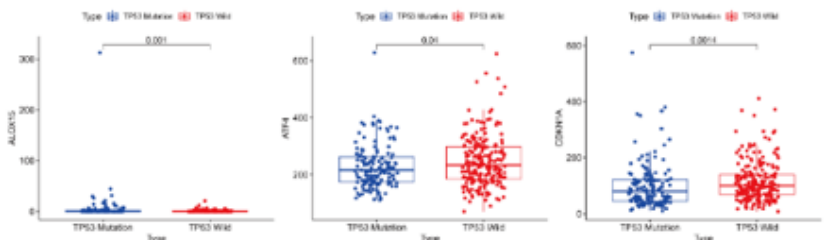

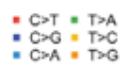
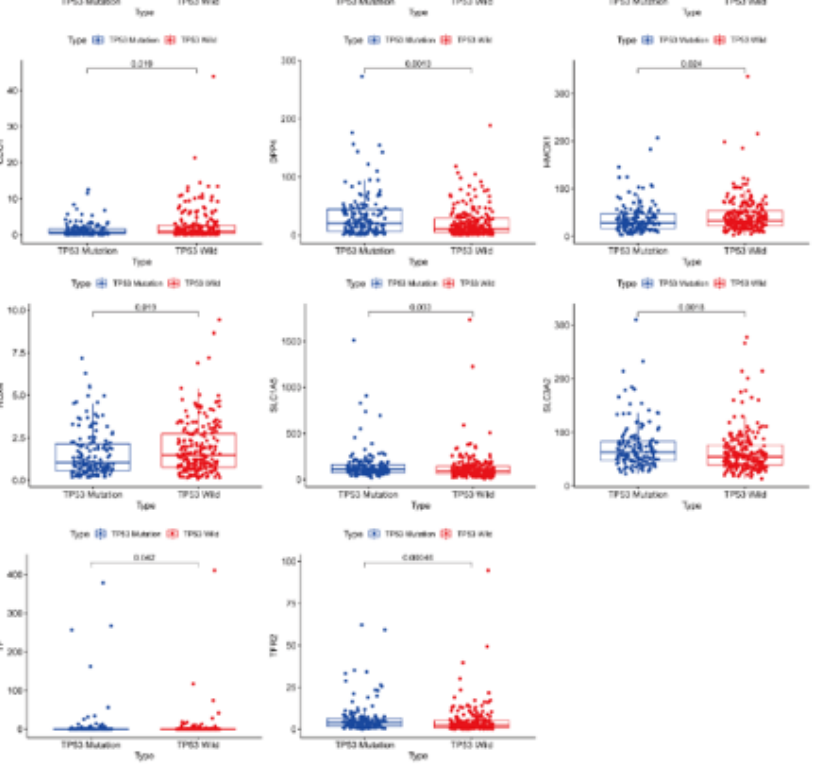

E

$\mathrm{D}$
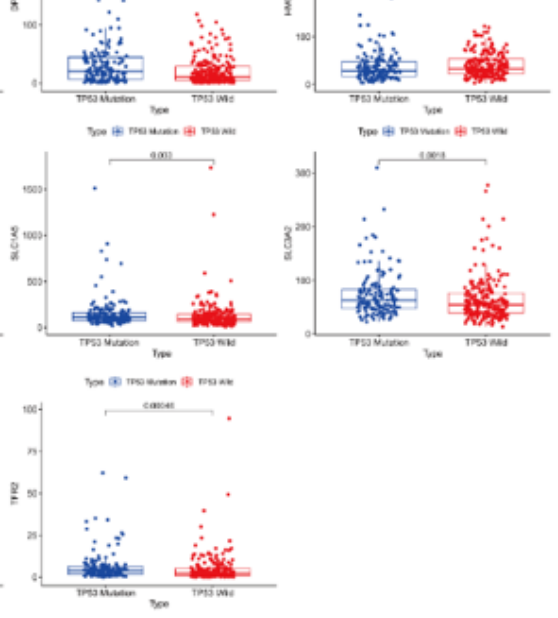

C
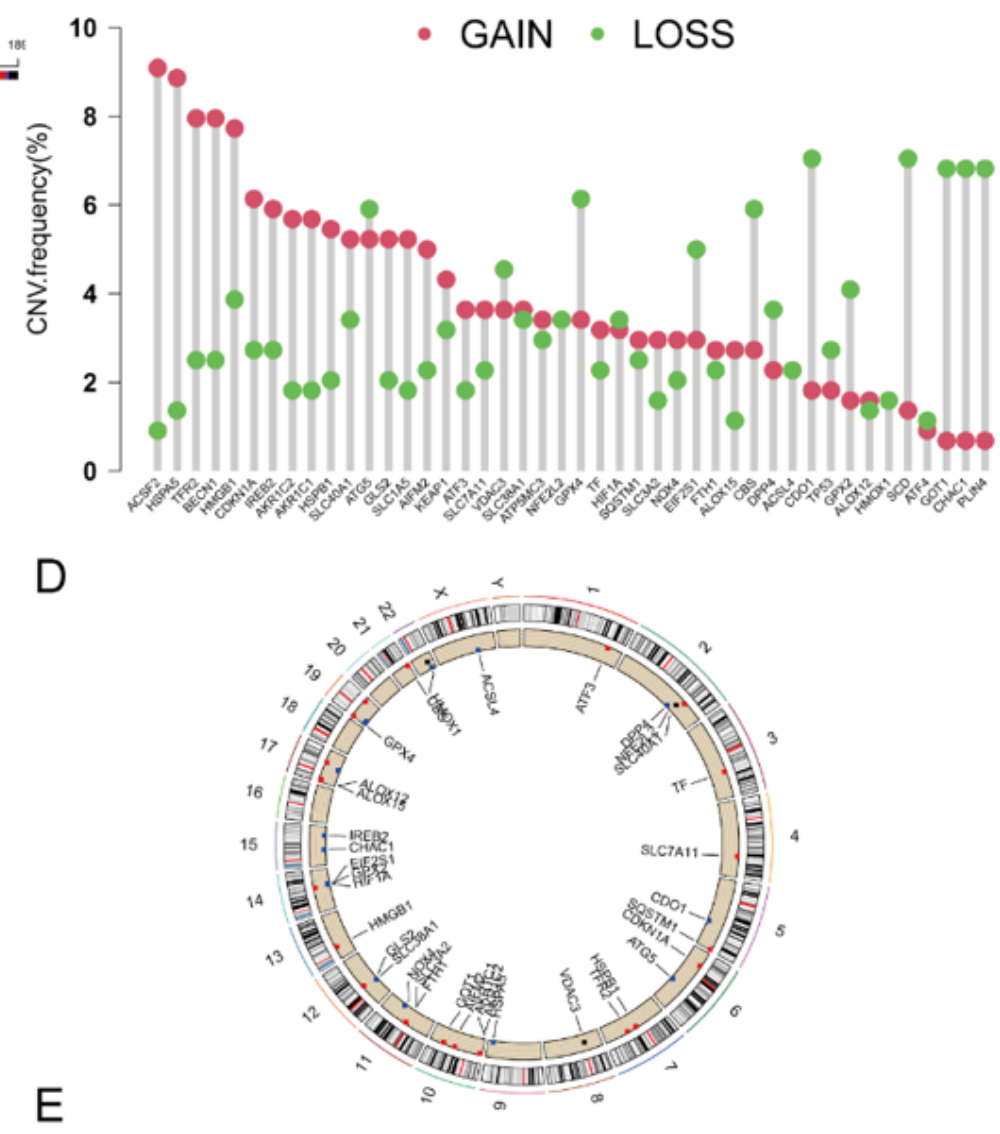

Type 追 Normal 追 Tumor

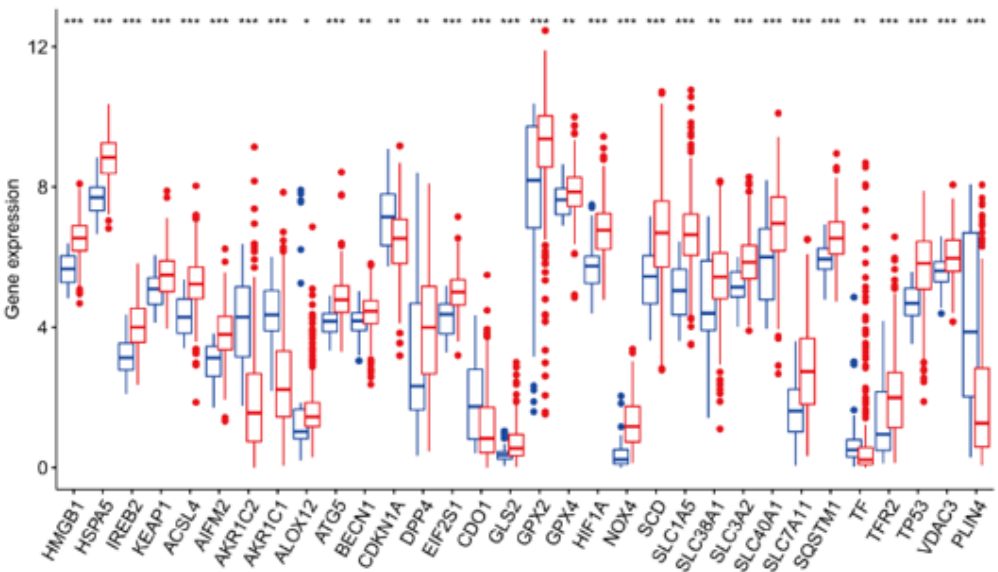

Figure 1

Genetic and transcriptional alterations of FRGs in GC. (A) Mutation frequencies of 44 FRGs in 433 patients with GC from the TCGA-STAD cohort. (B) The relationship between TP53 mutation and the expression level of FRGs in GC. (C) Frequencies of CNV gain and loss among FRGs. (D) Chromosome 23 CNV changes in FRGs. (E) Differences in the expression of FRGs between normal and GC tissues that are statistically significant. FRGs stand for ferroptosis-related genes; GC is for gastric cancer; STAD stands for stomach adenocarcinoma; TCGA stands for The Cancer Genome Atlas; and CNV stands for copy number variation. 
A

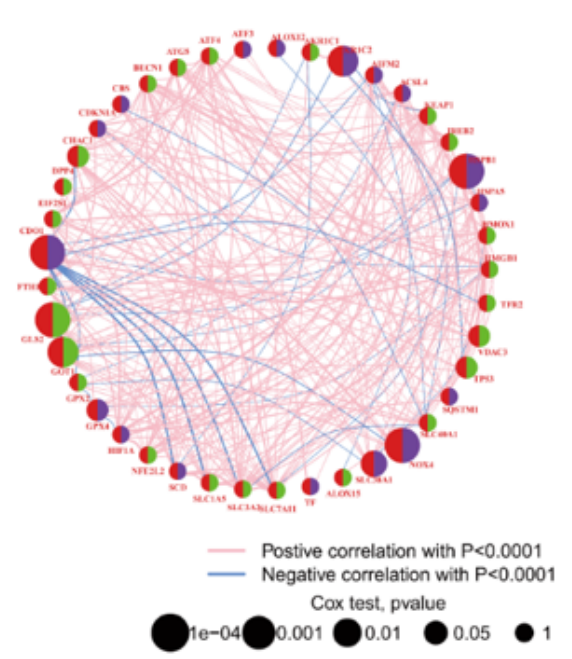

C

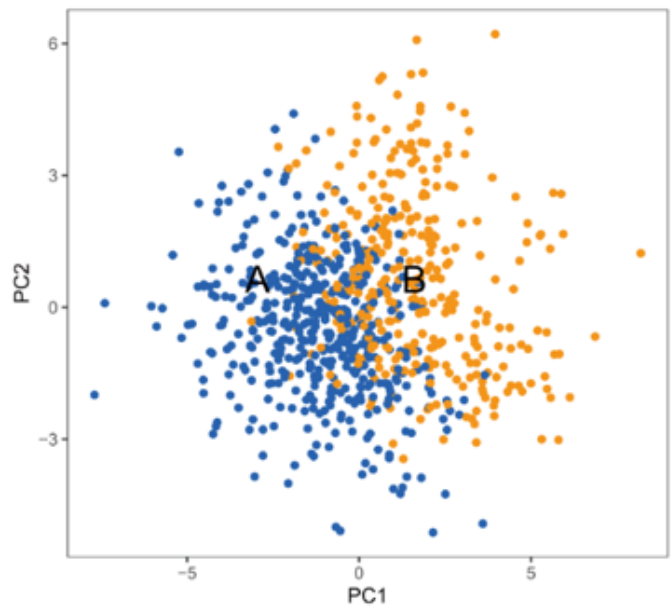

- Ferroptosis

B

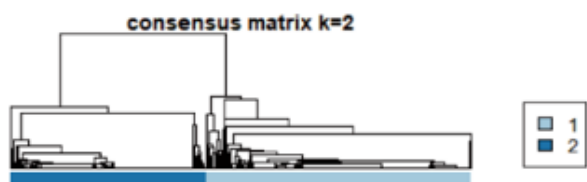

- Risk factors

- Favorable factors

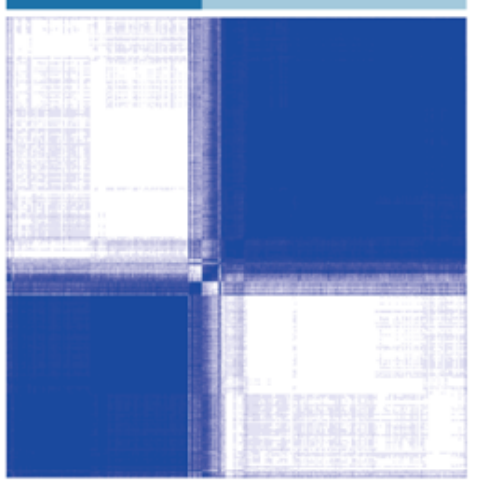

1
2

E
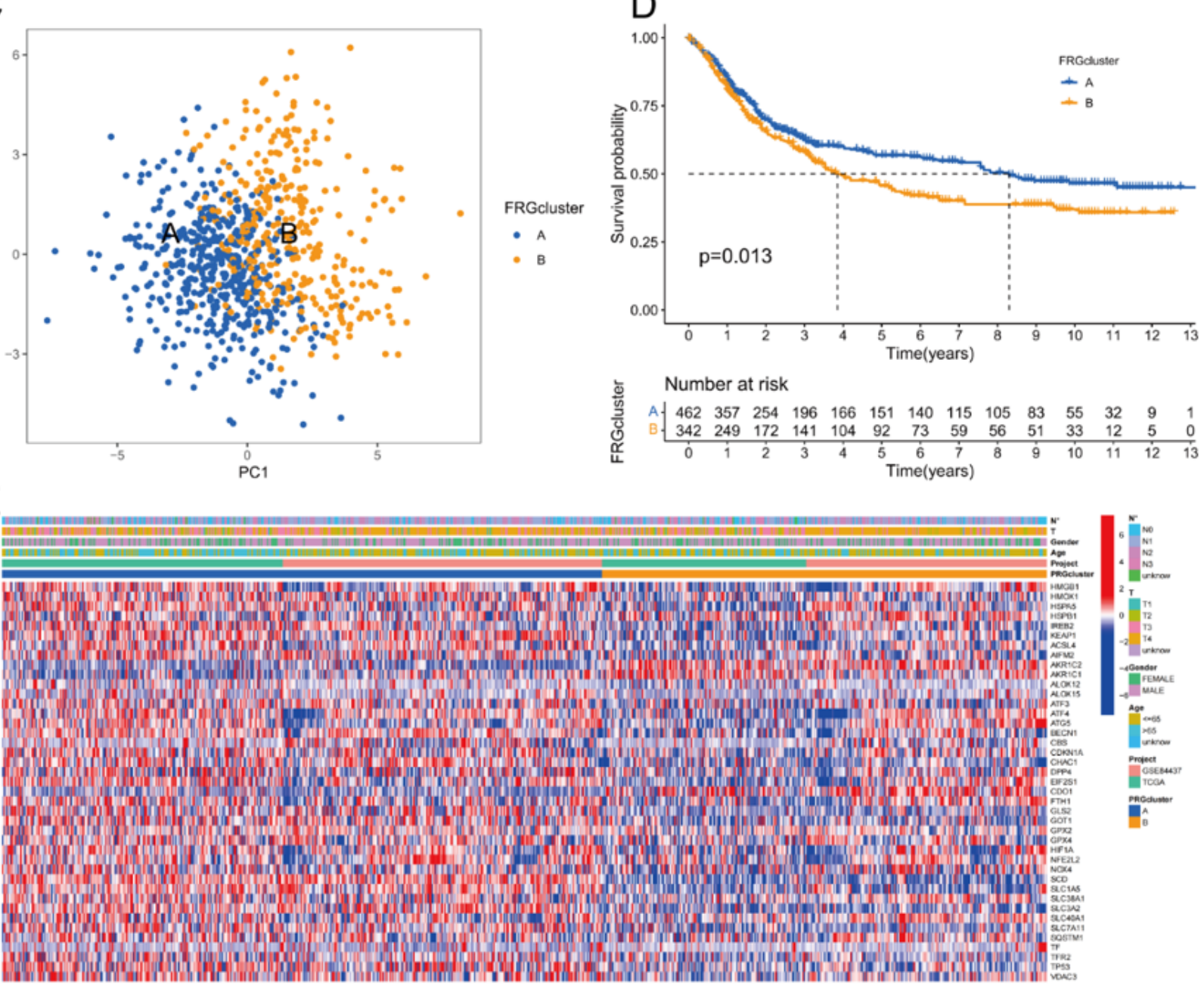

Figure 2

FRG subtypes and biological characteristics and clinicopathological characteristics of two different subtypes of samples. (A) Interaction between FRGs in GC. The lines connecting the FRGs represent their interactions, and the thickness of the lines represents the strength of the relationship between the FRGs. 
Red and blue represent positive correlations and negative correlations respectively. (B) Consensus matrix heatmaps of two clusters $(k=2)$ and their related regions. (C) PCA reflected huge differences in the transcriptome between the two subtypes. (D) Univariate analysis showing 44 FRGs associated with OS time. (E) Differences in clinicopathological features and FRG expression levels between two different subtypes. FRGs, ferroptosis-related genes; GC, gastric cancer; PCA, principal components analysis; OS, overall survival.

A $\quad$ FRGcluster 白 A 白 $_{\text {B }}$

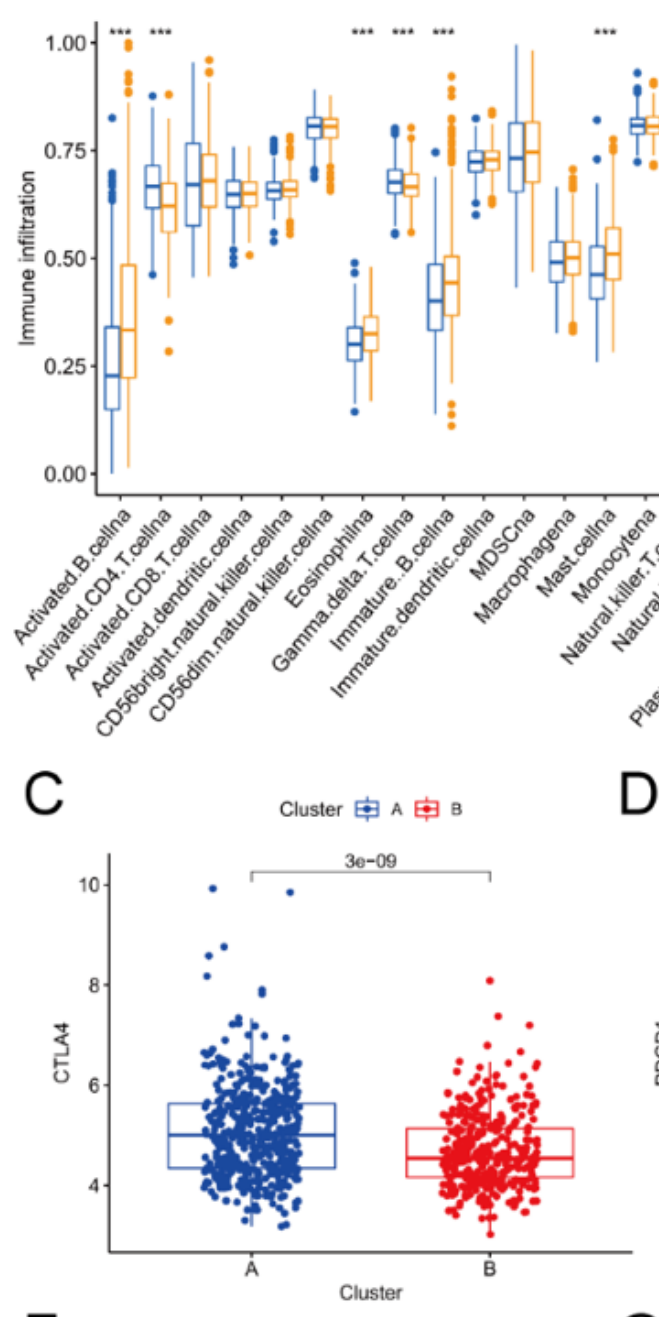

$\mathrm{F}$
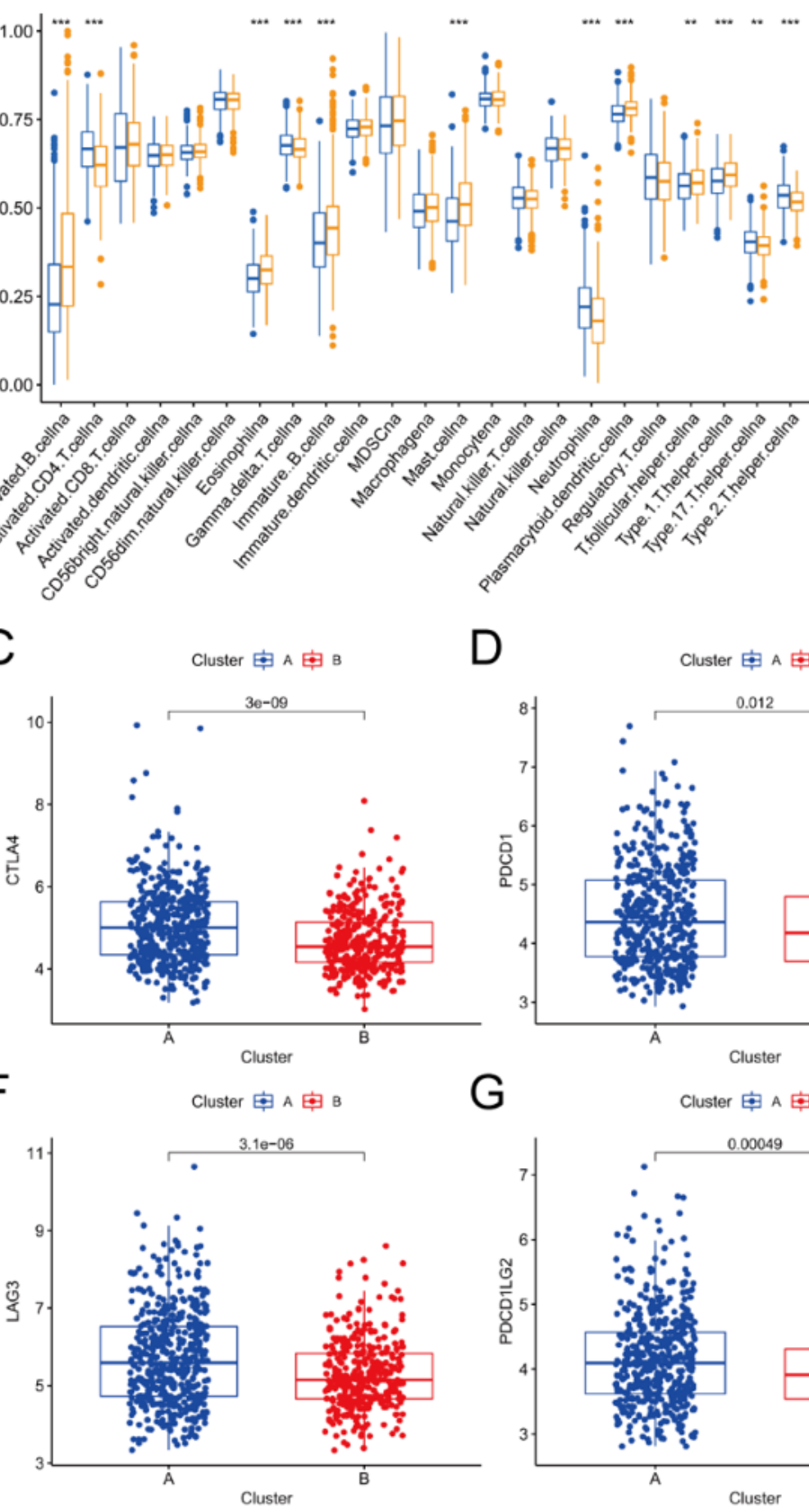

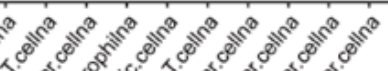

(5)

D

G
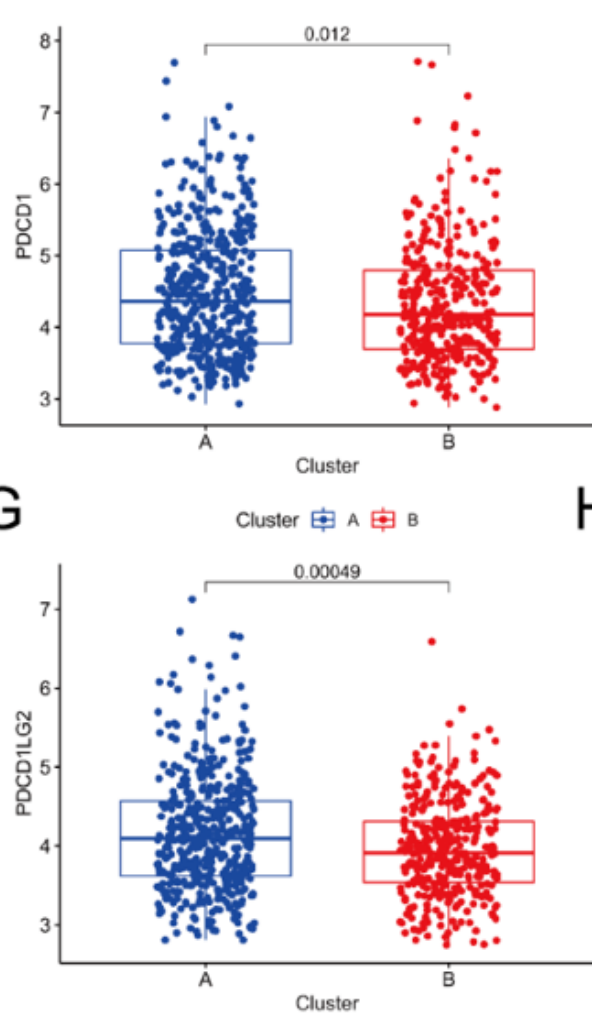

$\mathrm{H}$
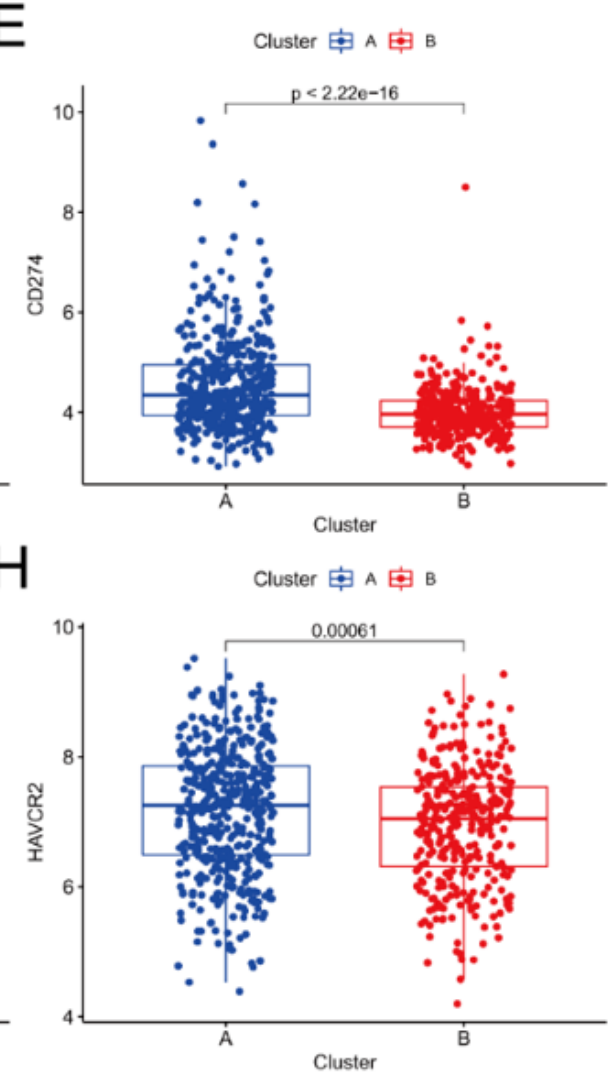


\section{Figure 3}

Correlations of tumor immune cell microenvironments and two GC subtypes. (A) Abundance of 23 infiltrating immune cell types in the two GC subtypes. (B) Correlation between two GC subtypes and TME score. (C) Expression levels of CTLA4, PDCD1, CD274, LAG3, PDCD1LG2 and HAVCR2 in the two GC subtypes. GC, gastric cancer; TME, tumor microenvironment. 

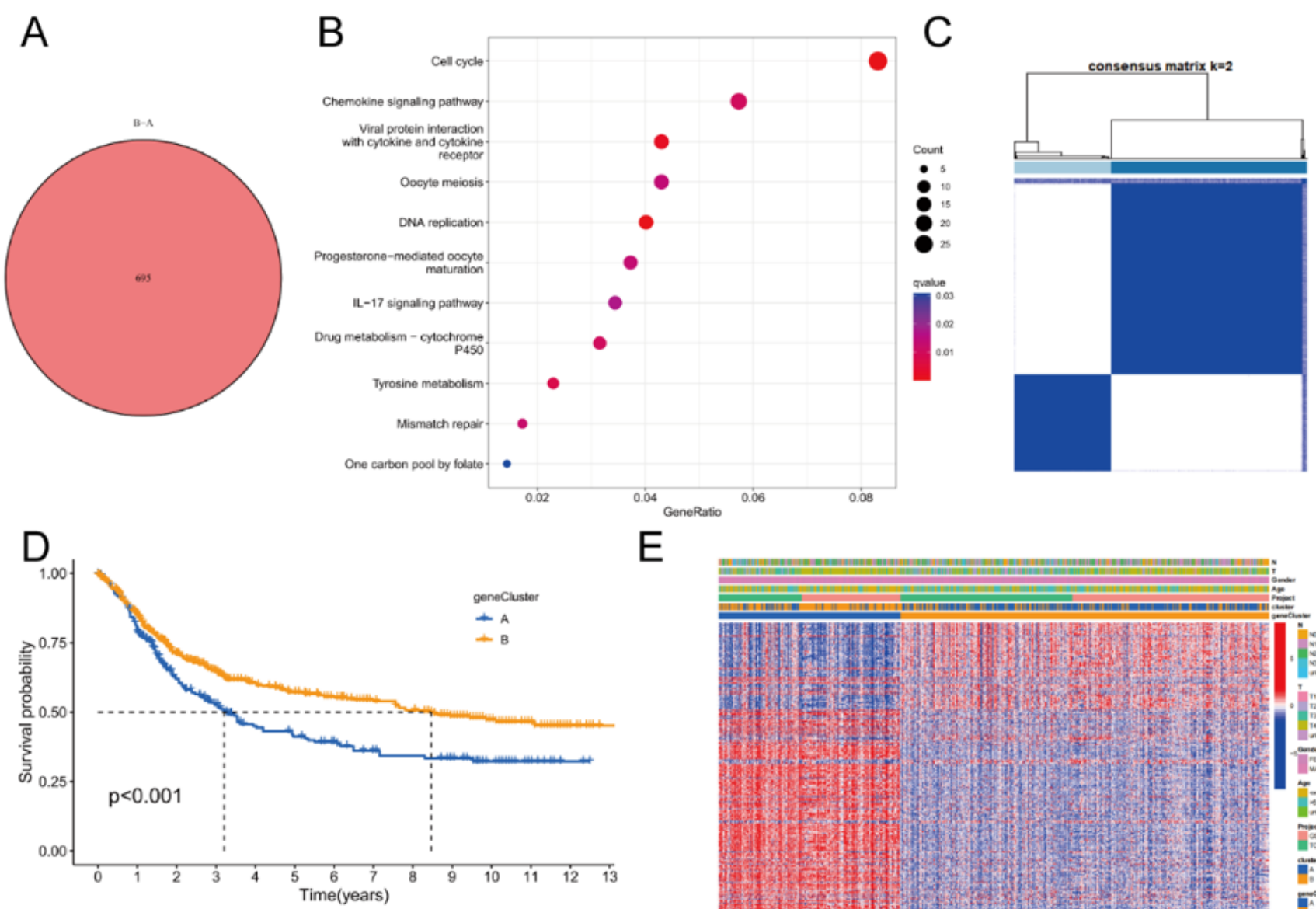

$\mathrm{E}$
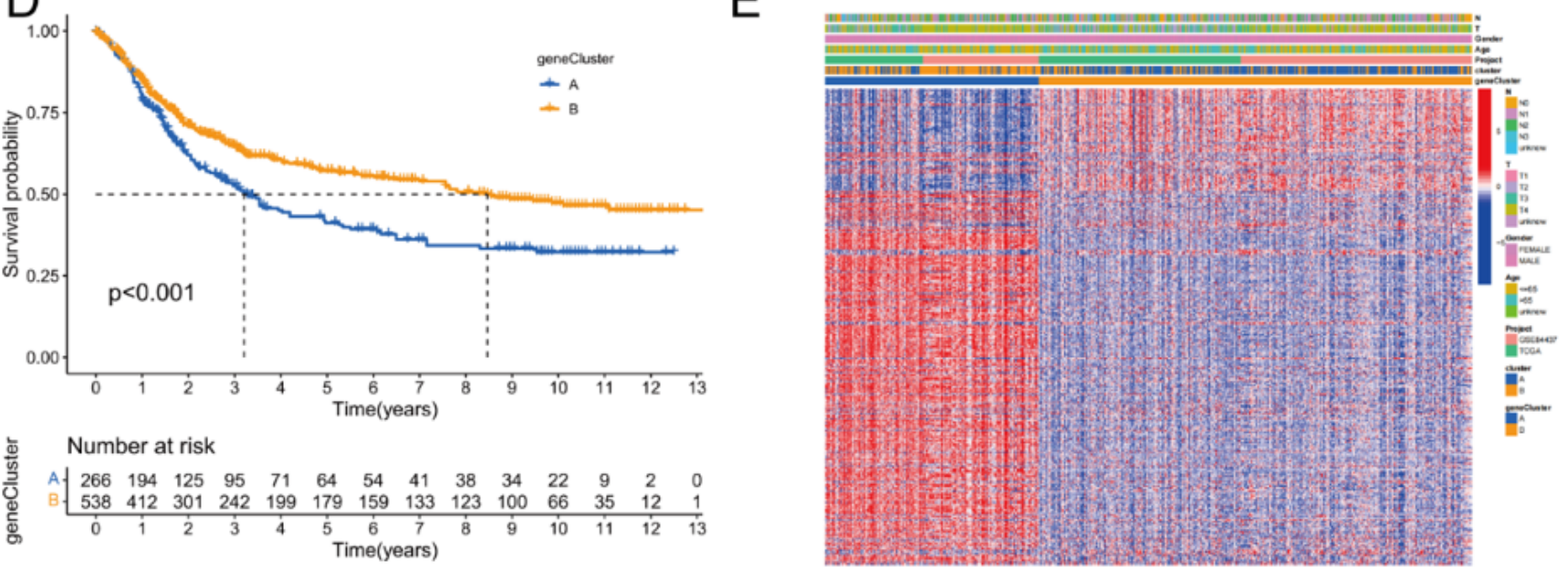

geneCluster 官 $\mathrm{A}$ 官 B

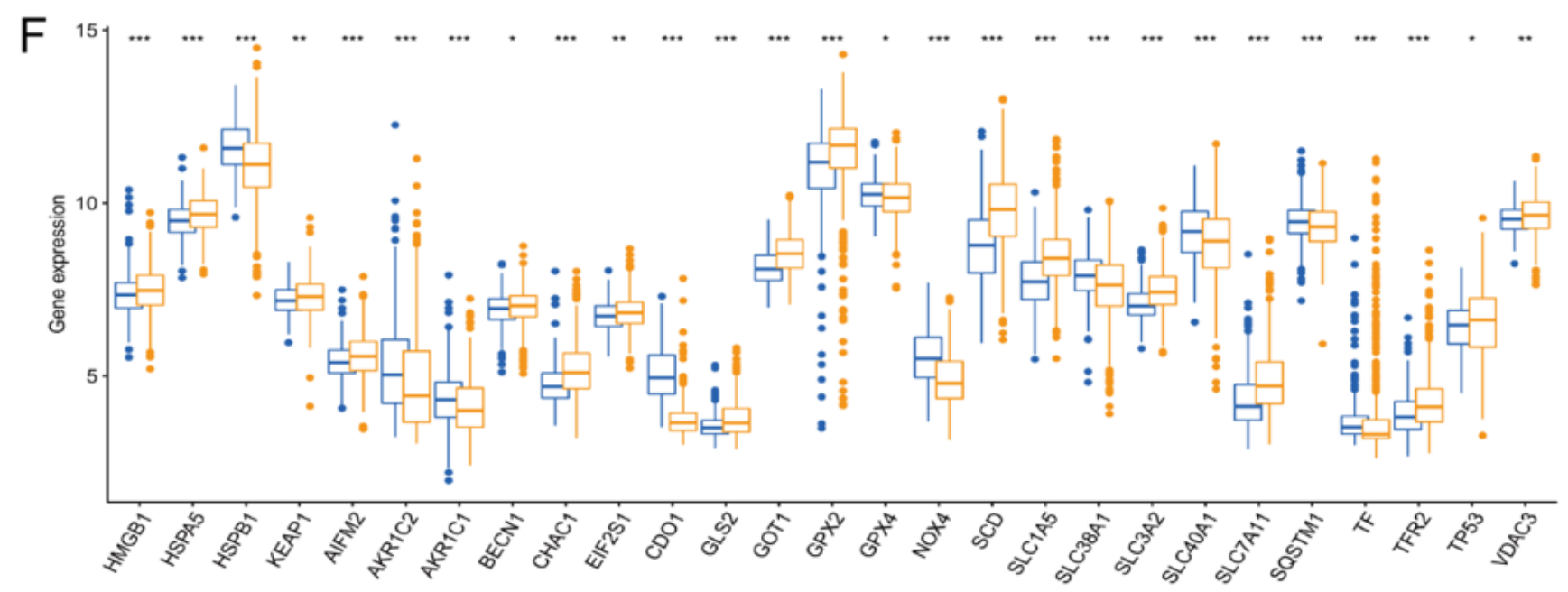

Figure 4

Gene subtype classification based on DEGs. (A) Venn diagram illustrating the number of genes that overlap in the two GC subtypes. (B) KEGG enrichment analysis of DEGs within and between two distinct classes. (C) Heatmaps of the consensus matrix for two clusters $(k=2)$ and their associated areas. (D) Kaplan-Meier curves illustrating the OS for the two gene subtypes (log-rank testing, p.001). (E) Association of clinicopathological characteristics with two gene subgroups. (F) Differences in FRG 
expression levels between the two gene subtypes. DEGs stand for differentially expressed genes; GC is for gastric cancer; KEGG stands for the Kyoto Encyclopedia of Genes and Genomes; OS stands for overall survival; and FRGs stands for ferroptosis-related genes.

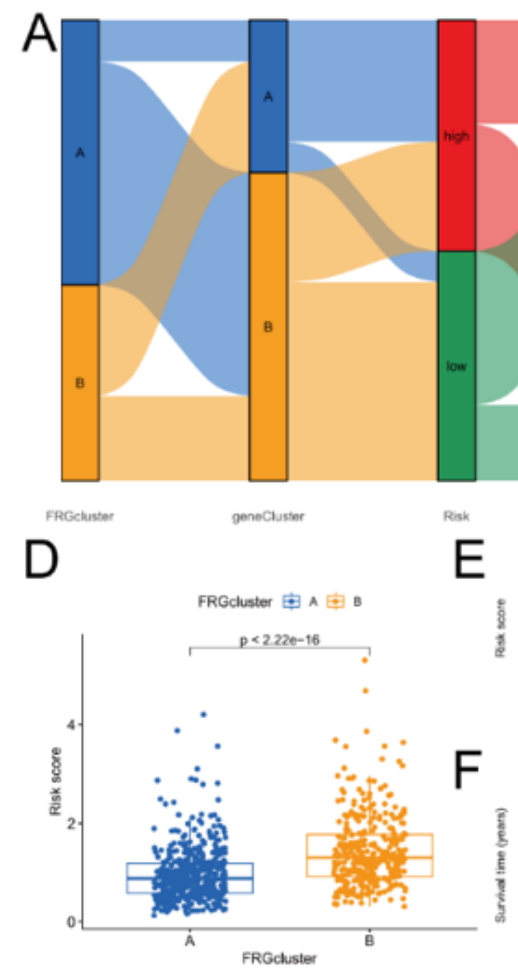

B
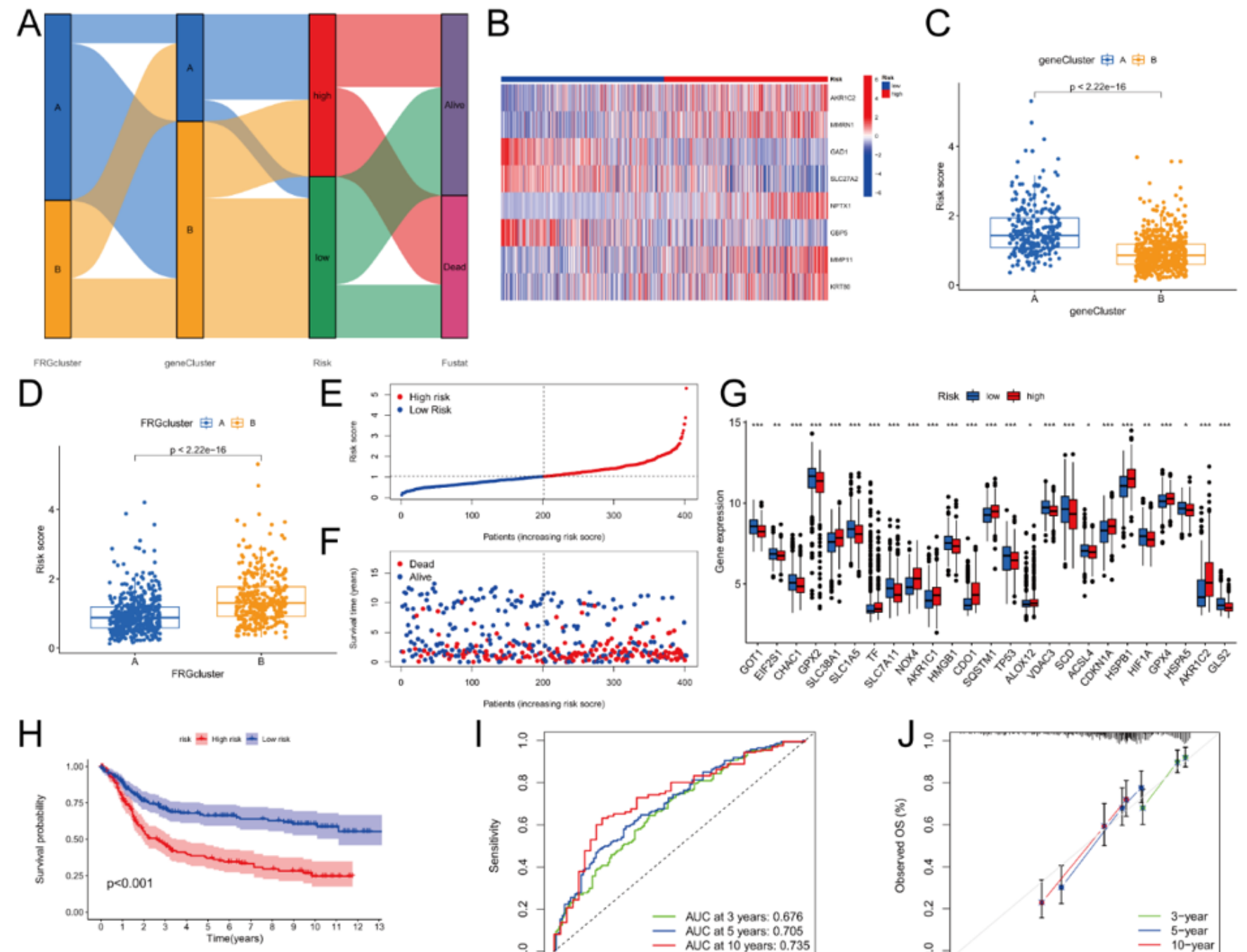

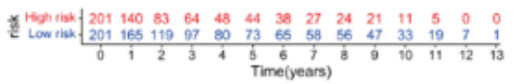

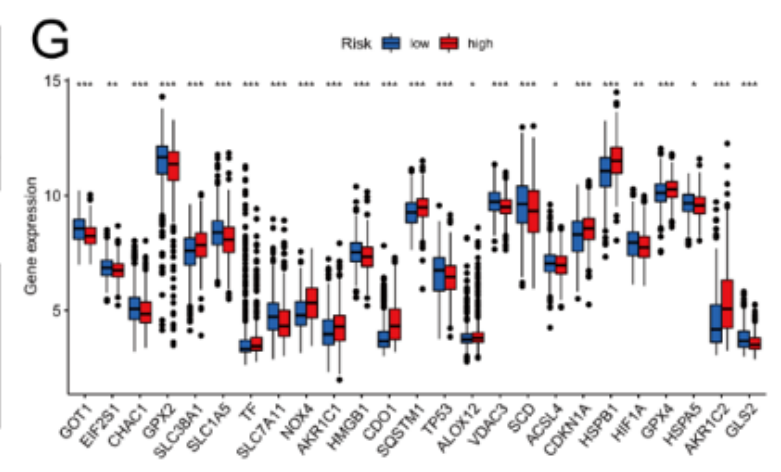

100
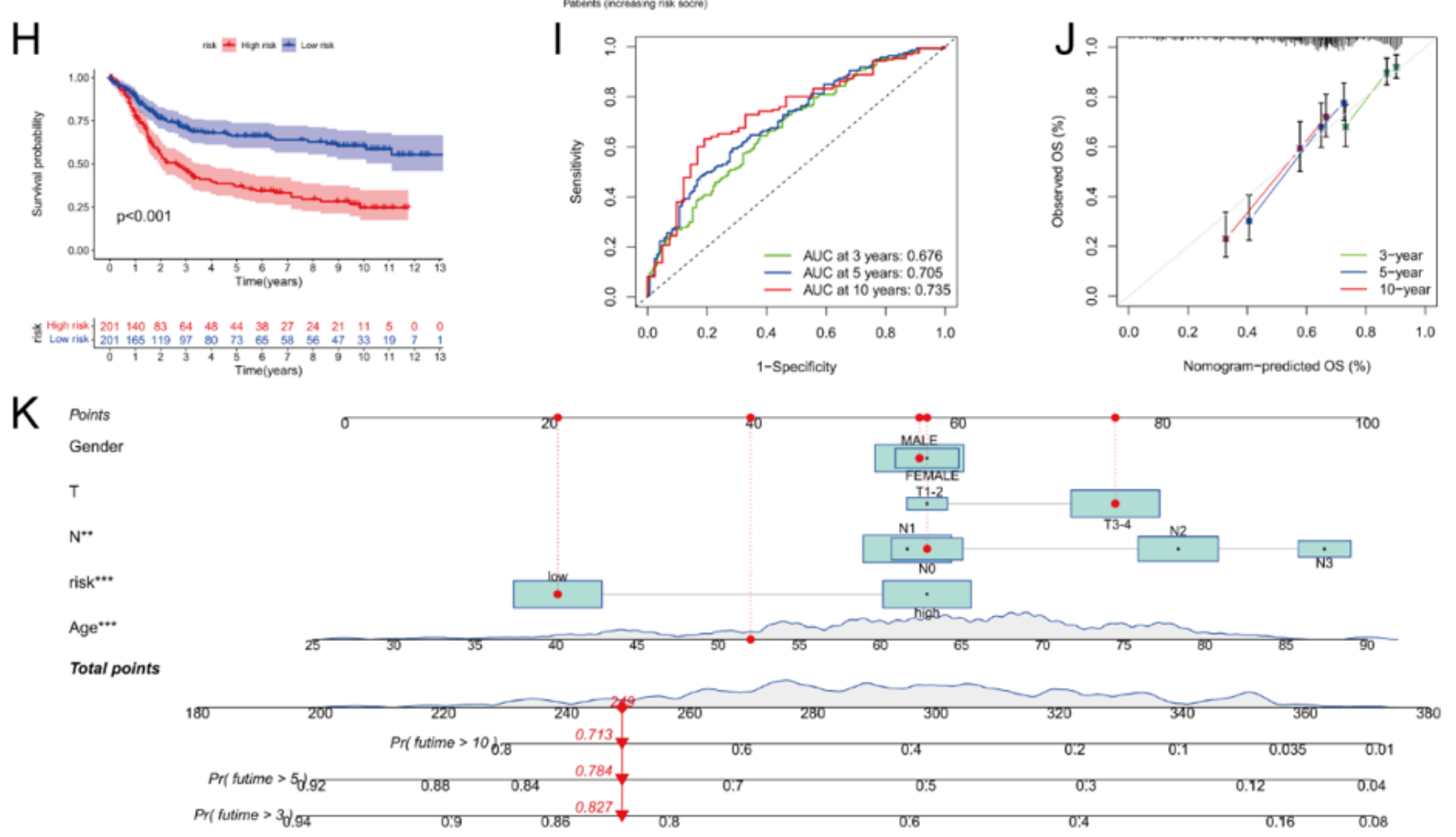

Figure 5 
The training set was adopted in the construction and validation of the risk-score. As seen in Figure 1, the alluvial diagram shows the distribution of subtypes across various risk-score and survival result groups. (B) Genes identified using a multivariate Cox regression analysis of genes related with OS. A comparison of the risk scores between gene and ferroptotic subtypes is shown in (C-D). (E-F) Rank-and-scatter plots illustrating the distribution of risk scores and the survival status of patients. (G) Variations in the expression of FRGs between the two groups. Comparison of survival between high-risk and low-risk groups using the Kaplan-Meier method $(\mathrm{H})$. (I) Receiver operating characteristic (ROC) curves to estimate the sensitivity and specificity of three-, five-, and ten-year survival based on the r risk-score. (J) Calibration curves of the nomogram for estimating three-, five-, and ten-year OS in the training sets. (K) Nomogram for predicting the three-, five-, and ten-year OS of GC patients in the training set. FRGs, OS, overall survival; ferroptosis-related genes; ROC, receiver operating characteristic; GC, gastric cancer. 

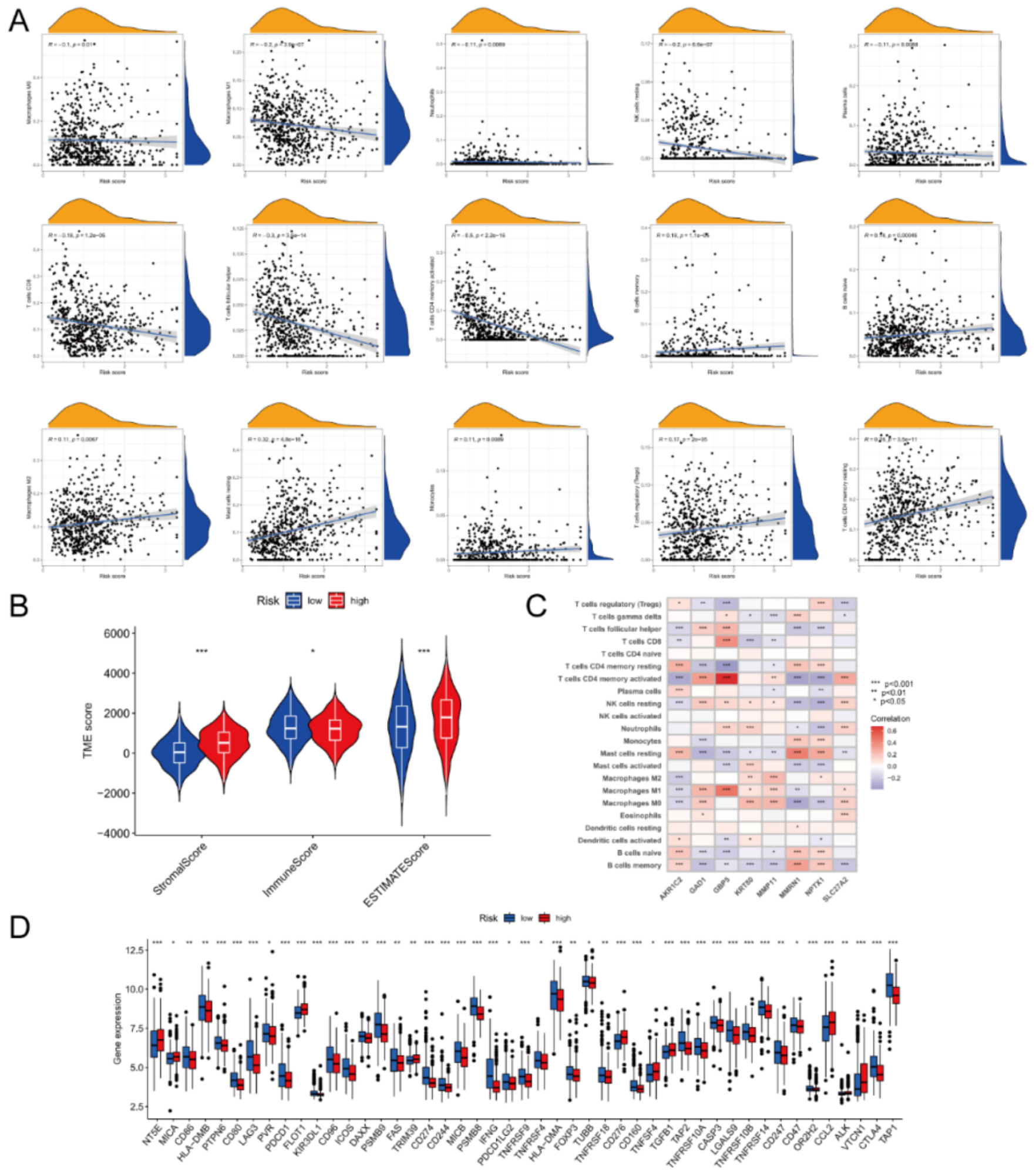

Figure 6

Compare and contrast the TME and checkpoints in the high- and low-risk categories. (A) Risk-score correlations with immune cell types. (B) The suggested model's relationships between immune cell abundance and eight genes. (C) The suggested model's relationships between immune cell abundance and eight genes. (D) Expression of immune checkpoints in the two groups. TME, tumor microenvironment. 


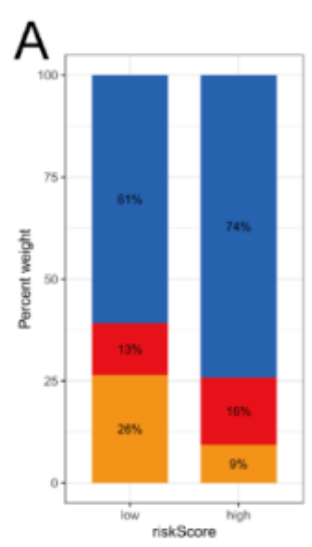

E
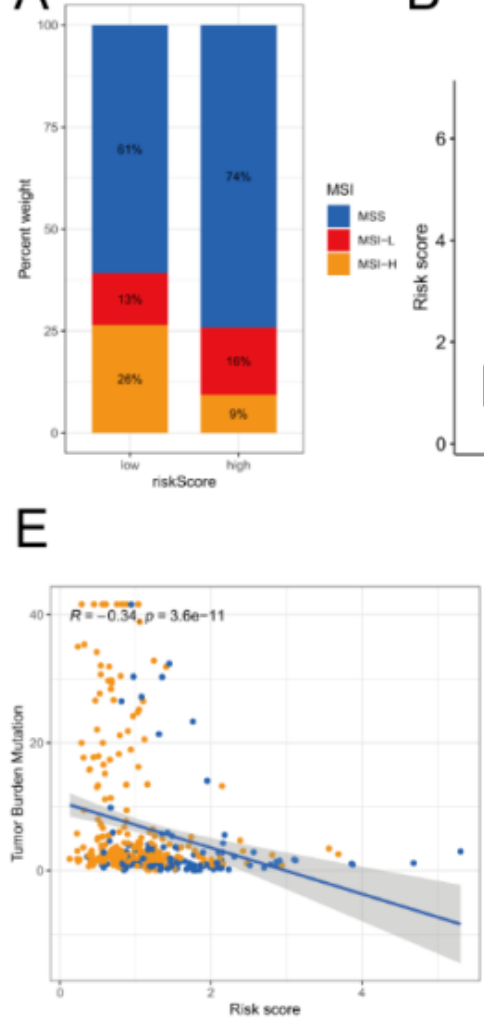

B

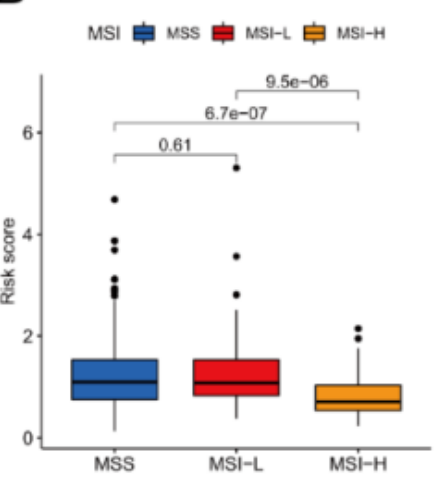

C

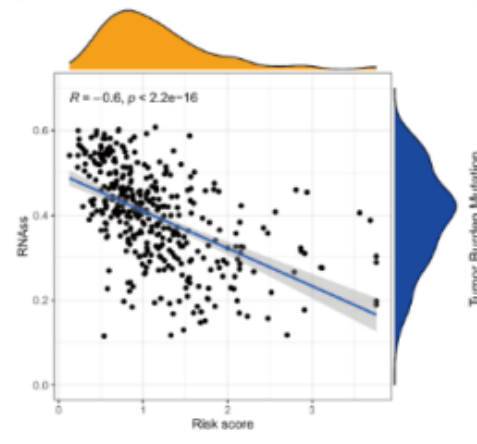

D

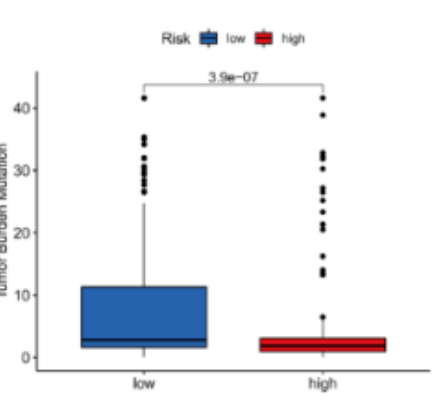

\section{$G$}
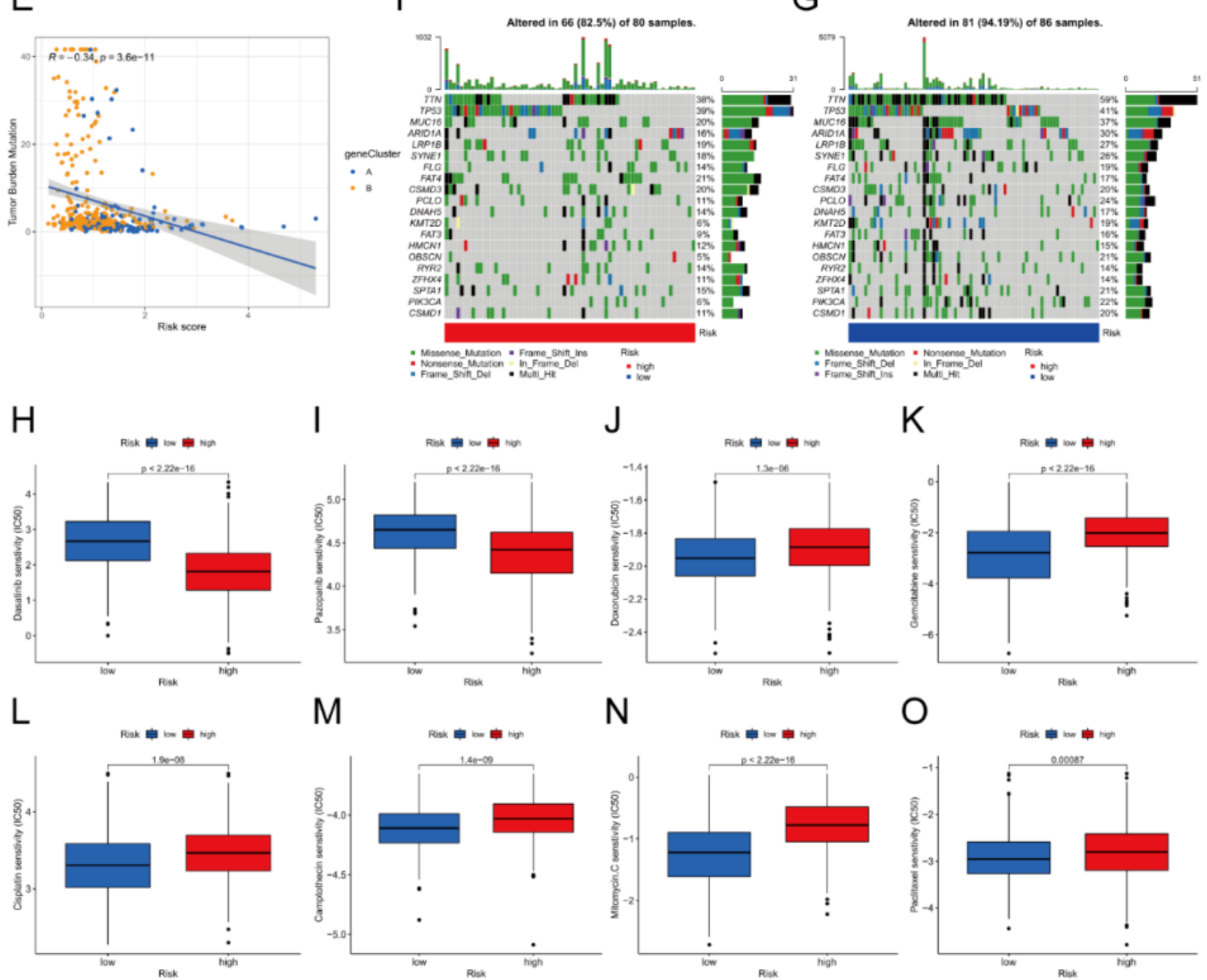

$\mathrm{N}$
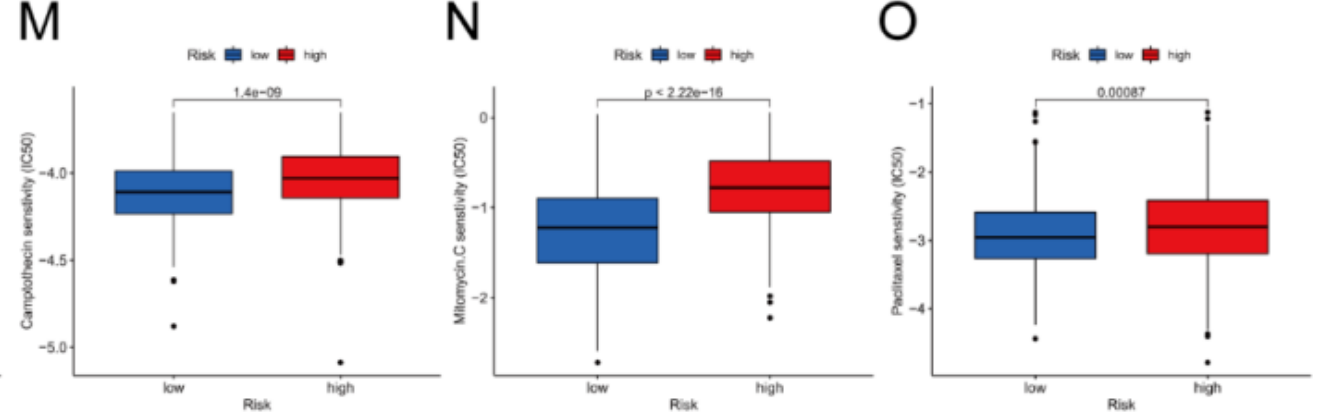

Figure 7

In-depth examination of the risk-score in GC. (A-B) Relationships between risk-score and MSI. (C) Relationships between risk-score and CSC index. (D) TMB in diverse risk-score groups. (E) Spearman correlation analysis of the risk-score and the total number of occurrences. (F-G) Somatic mutation 
features were shown as a waterfall plot using high and low FRG-scores, respectively. In this table, each column represents a different patient. TMB is shown in the bar chart above, and the numbers on the right reflect the frequency with which each gene has been mutated. The proportions of each variant type are illustrated by the bar chart on the right. H-O Relationships between risk-score and chemotherapeutic sensitivity in cancer patients GC stands for gastric cancer; MSI stands for microsatellite instability; CSC is for cancer stem cell; and TMB stands for tumor mutation burden.

\section{Supplementary Files}

This is a list of supplementary files associated with this preprint. Click to download.

- SupplementaryTables1.xls

- SupplementaryTableS2.xls

- SupplementaryTableS3.xls

- SupplementaryTableS4.xls

- SupplementaryTables5.xls

- SupplementaryTableS6.xls

- SupplementaryTables7.xls

- SupplementaryTableS8.xls

- SupplementaryTables9.xls

- SupplementaryTableS10.xls

- FigureS1.png 